\title{
HIGHLIGHTS
}

BONE

\section{Could IFN- $\gamma$ be a new therapeutic target in osteoporosis?}

Data from studies in mice, published in the Journal of Bone and Mineral Research, shed light on the role of interferon (IFN) $-\gamma$ in bone formation and suggest that this cytokine could be a new therapeutic target in osteoporosis.

\section{4 ...the reduced lumbar spine BMD seen in OVX mice was corrected by IFN- $\gamma$ treatment... 77}

Although the function of IFN- $\gamma$ in osteoclast biology has been studied previously, its role in osteoblast development and function, and therefore in bone remodelling as a whole, are not well understood. To investigate this role, Duque et al. obtained and analyzed IFN- $\gamma$ receptor knockout $\left(I F N \gamma R 1^{-/}\right)$mice, in which IFN- $\gamma$ signalling is blocked.

Bone volume was reduced by $45 \%$ in $I F N \gamma R 1^{-/-}$mice in comparison with IFN $\gamma R 1^{+/+}$mice and this discrepancy was associated with reduced trabecular and cortical thickness, which are changes characteristic of osteoporosis. In addition, rates of bone formation and mineral apposition, bone cellularity (numbers of osteoblasts and osteoclasts) and circulating levels of markers of bone formation and bone resorption were reduced in $I F N \gamma R 1^{-/-}$mice in comparison with their IFN $\gamma R 1^{+/+}$littermates.

Next, the researchers studied the effects of IFN- $\gamma$ administration on bone structure, strength and cellularity in ovariectomized (OVX) mice-a model of osteoporosisand sham ovariectomized (SHAM) mice. "The SHAM model was used to see if IFN- $\gamma$ could induce bone formation in relatively old mice and the OVX model was used to test whether IFN- $\gamma$ was strong enough to rescue mice from osteoporosis," explains Gustavo Duque, the corresponding author on the paper.

In 10-week-old SHAM mice, 6 weeks of IFN- $\gamma$ treatment $(10,000$ IU, three times per week) resulted in increased lumbar spine bone mineral density (BMD) in comparison with control (vehicle only) treatment, and the reduced lumbar spine BMD seen in OVX mice was corrected by IFN- $\gamma$ treatment. Bone volume, strength, and microarchitecture improved, bone cellularity increased, and mineral apposition rates were higher in SHAM and OVX mice treated with IFN- $\gamma$ than in mice in the control groups.

"The most significant finding is that IFN- $\gamma$ acts as an anabolic agent in bone," states Duque. "Considering that most current osteoporosis treatments are antiresorptives and that the most important mechanism of osteoporosis in older individuals is lack of bone formation, we think that a new anabolic treatment for osteoporosis is highly needed."

Jenny Buckland

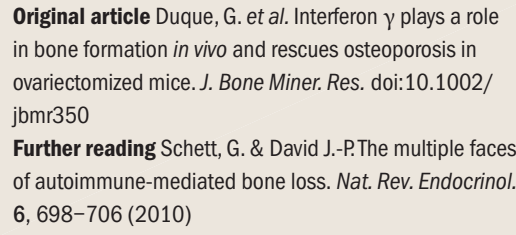

Original article Duque, G. et al. Interferon $\gamma$ plays a role in bone formation in vivo and rescues osteoporosis in ovariectomized mice. J. Bone Miner. Res. doi:10.1002/ jbmr350

Further reading Schett, G. \& David J.-P.The multiple faces of autoimmune-mediated bone loss. Nat. Rev. Endocrinol. 6, 698-706 (2010) 